\title{
Influence of Wind Forcing on Modulation and Breaking of One-Dimensional Deep-Water Wave Groups
}

\author{
Alina Galchenko, Alexander V. Babanin, And Dmitry Chalikov \\ Swinburne University of Technology, Melbourne, Victoria, Australia \\ I. R. YOUNG \\ Australian National University, Canberra, Australian Capital Territory, Australia \\ BRIAN K. HAUS \\ University of Miami, Coral Gables, Florida
}

(Manuscript received 12 April 2011, in final form 14 October 2011)

\begin{abstract}
Evolution of nonlinear wave groups to breaking under wind forcing was studied by means of a fully nonlinear numerical model and in a laboratory experiment. Dependence of distance to breaking and modulation depth (height ratio of the highest and the lowest waves in a group) on wind forcing was described. It was shown that in the presence of a certain wind forcing both distance to breaking and modulation depth decrease; the latter signifies slowing down of the instability growth. It was also shown that wind forcing significantly reduces the energy loss in a single breaking event.
\end{abstract}

\section{Introduction}

Wave breaking is of interest in oceanography because it plays an important role in exchange of energy between atmosphere and ocean, in the air-sea exchange of momentum, mass, and heat. Breaking is the main sink of wave energy; it limits the wave growth. Wave breaking contributes to ocean acoustics and is also of significant importance for ocean remote sensing, coastal engineering, navigation, and other applications (for a review, see, e.g., Babanin 2009). Dissipation due to breaking is defined by breaking probability (frequency of breaking occurrence; e.g., Babanin et al. 2001, 2007b; Cavaleri et al. 2007) and breaking severity (energy lost in a single breaking event; e.g., Manasseh et al. 2006; Babanin 2009; Babanin et al. 2010). The latter can vary over a very wide range (Galchenko et al. 2010).

It has been shown that there are two important characteristics of a wave group: lifetime and modulation

Corresponding author address: Alina Galchenko, Swinburne University of Technology, P.O. Box 218, Hawthorn VIC 3122, Australia.

E-mail: a08091985@gmail.com depth (Galchenko et al. 2010). Lifetime is time between the moment the wave is created and the moment when it breaks. This parameter is used in laboratory experiments and numerical simulations, rather than in the field; however, knowing lifetime, one can estimate breaking probability. The wave modulation depth $R$ is a height ratio of the highest $H_{h}$ and the lowest $H_{l}$ waves in the group, $R=H_{h} / H_{l}$ (Babanin et al. 2010). In Galchenko et al. (2010), it was shown that these parameters depend on initial primary wave steepness and ratio of initial steepnesses of the primary wave and the sideband. It was found that the severity of breaking grows with modulation depth for the values of the latter $1<R<5.5$. It was also shown that probability of breaking for wave groups with $R<2.2$ is very low.

Modulation depth is an indicator of the rate of instability growth. One of the questions discussed in terms of wave evolution and breaking is the frequency of occurrence of Benjamin-Feir instability and how often it can be the reason for breaking. Benjamin-Feir instability is not a rare event. In one-dimensional trains, it is always present, even for infinitesimally small waves. In two-dimensional wave fields, its activity is limited, but within the limits it is also always present. In both 
one-dimensional and two-dimensional cases, BenjaminFeir instability may lead to a freak wave or may result in wave breaking (there is also the third option: full recurrence of the wave train without breaking). The main factor that defines what event is the most probable is the wave steepness, because the rate of instability depends on the steepness (provided that the bandwidth in frequency and angle is the same). For low steepness, the Benjamin-Feir instability will lead a uniform wave train to an occurrence of a wave higher than average (but that wave will only be marginally higher) and then to a full recurrence of the original uniform wave train. For steep waves, the growth rate will be large and lead to breaking. Also, there is a range of steepnesses when waves grow very high because of Benjamin-Feir instability but do not break, and this is when freak waves are most probable. In Babanin et al. (2011b), this range was identified as $a k=0.11-0.13$. Freak waves, however, are rare events, whereas wave breaking is frequent. There are a number of other mechanisms apart from BenjaminFeir instability that may lead to wave breaking. In deep water and in the absence of environmental forcing (or when the forcing is relatively weak), there is only one such mechanism, and it is linear superposition. However, Babanin et al. (2010, 2011a) conclude on the basis of multiple direct and indirect evidences, that, although linear superposition is theoretically capable to lead to a breaking in the ocean, in practice it is a very rare occurrence, and the main breaking mechanism in typical oceanic conditions is the Benjamin-Feir instability.

Benjamin-Feir instability in two-dimensional wave fields has been studied by means of dynamic equations (e.g., McLean 1982a,b; Mori et al. 2011), phase average equations (e.g., Alber 1978), and experimentally (Onorato et al. 2009a,b; Waseda et al. 2009). All the studies show that it exists in two-dimensional wave fields, but within some limited range of directional spreadings. Babanin et al. (2010) suggested that this range depends on combination of the directional bandwidth and mean wave steepness; that is, if the steepness is higher, the directional spreading can also be broader while the instability is still active. Babanin et al. (2011a,b) have investigated this range quantitatively in a laboratory experiment in two-dimensional wave tank. They concluded that, for directional spreading and wave steepness typical for oceanic conditions, Benjamin-Feir instability is still active and leads to wave breaking.

Wind forcing has many effects on the evolution of nonlinear wave groups to breaking. Wind affects the rate of instability growth (Trulsen and Dysthe 1992; Waseda and Tulin 1999; Babanin et al. 2010). Because this rate affects the wave-breaking severity, wind therefore can be an important player in the context of the breaking strength and whitecapping dissipation. The wind forcing enhances breaking probability (e.g., Hwang et al. 1989; Banner et al. 2000; Babanin et al 2001, 2007b) but reduces the breaking severity (Babanin et al. 2010). In the coupled wind-wave system, the breaking severity, in turn, affects the wind-to-wave energy and momentum input, even if locally (Babanin et al. 2007a). It is essential to note, however, that capacity of the wind to modify the condition of breaking onset is marginal, unless the wind is very strong (Babanin 2009; Babanin et al. 2010; Toffoli et al. 2010).

In this paper, we are pursuing two main questions that need an answer. First is how wind forcing changes the dependencies described in Galchenko et al. (2010): that is, the behavior of lifetime and modulation depth as functions of initial steepness and the dependence of breaking severity on modulation depth. The investigations necessary for estimating lifetime and for describing the evolution of modulation depth were conducted by means of fully nonlinear Chalikov-Sheinin (CS) model described in Chalikov and Sheinin (2005). The CS model implemented in this study was coupled with the atmospheric boundary layer (Chalikov and Rainchik 2011).

An important advantage of the CS wave model is that it does not have limitations on steepness and the duration of propagation; it does not accumulate numerical errors. As mentioned above, for studying wind-wave interaction, the CS model is coupled with a wave boundary layer model (Chalikov and Rainchik 2011). In this coupled wind-wave model, waves are the object of the modeling. Equations for the boundary layer are solved along with full potential wave equations, and the solutions for air and water are matched through the interface. The fully nonlinear model allows describing the effects of the wave crest sharpening, which strongly increases the pressure anomalies. It is important that the model works in physical rather than Fourier space. This is an apparent advantage of the model, because many processes (e.g., group effects) can be observed only in physical space. Energy input to waves is also best described in physical rather than Fourier space because of local steepness effects (e.g., Donelan et al. 2005, 2006; Babanin et al. 2007a; Savelyev et al. 2011).

The second question is how the severity of a single breaking event changes with wind forcing. As mentioned above, in response to the wind forcing, the breaking probability grows, whereas the breaking severity reduces (Babanin 2009; Galchenko et al. 2010). As a result, a trend of such an important feature as the whitecapping dissipation is not apparent.

Although field investigations of the breaking severity have been an active topic of research lately, there is a clear lack of studies dealing with influence of wind on 
the breaking severity as such. Field measurements of breaking severity are rather complicated. Several studies (e.g., Monahan 1971; Zhao and Toba 2001; Guan et al. 2007; Yuan et al. 2009) established a dependence between wind speed and whitecap coverage. According to these studies, in most cases whitecap coverage tends to increase with wind speed (although data scatter is very significant). However, whitecap coverage depends not only on the breaking severity but also on breaking probability and the physical and chemical properties of the water (e.g., Wu 2000; Stramska and Petelski 2003). Thus, it is impossible to estimate the breaking strength of a single event based on the information about whitecap coverage.

A few experiments, both in laboratory and in the field, have been made with the purpose of estimating the sizes and penetration depths of bubbles produced by breakers. It is supposed that larger bubbles correspond to more severe breakers (Stolte 1992; Manasseh et al. 2006; Babanin 2009). Most of the studies show that the size of the bubbles and depth of their penetration into the water grow with wind forcing. However, just as in case with whitecap coverage, single breaking events are not always separated. Thus, the influence of wind on breaking severity is still in need of detailed insights. In the present paper, a laboratory experiment aimed at studying breaking severity of wave groups with wind forcing is described. This is the limiting case of one-dimensional (long crested) waves, and two-dimensional issues of modulational instability, wave breaking, and wind forcing have to be studied separately.

\section{Benjamin-Feir instability in the presence of wind}

The wave system in question is initially assigned by a superposition of two sinusoidal signals with different amplitudes and close wavenumbers: a carrier wave with a steepness $\varepsilon_{1}=a_{1} k_{1}$ and a sideband with a steepness $\varepsilon_{2}=a_{2} k_{2}$, where $a_{1}$ and $a_{2}$ are the wave amplitudes and $k_{1}$ and $k_{2}$ are the wavenumbers. In the nondimensional model, however, the wave amplitude is not an independent parameter and is defined by the choice of wave steepness and wavenumber. Wavenumbers and therefore the initial bandwidth defined as

$$
\nu=\frac{2\left(k_{2}-k_{1}\right)}{k_{2}+k_{1}}
$$

in our numerical and laboratory experiments remain the same. Initial number of waves in a group is around $N=7$. In the nondimensional model, wavenumbers do not signify any particular dimensional values of wavenumbers; only
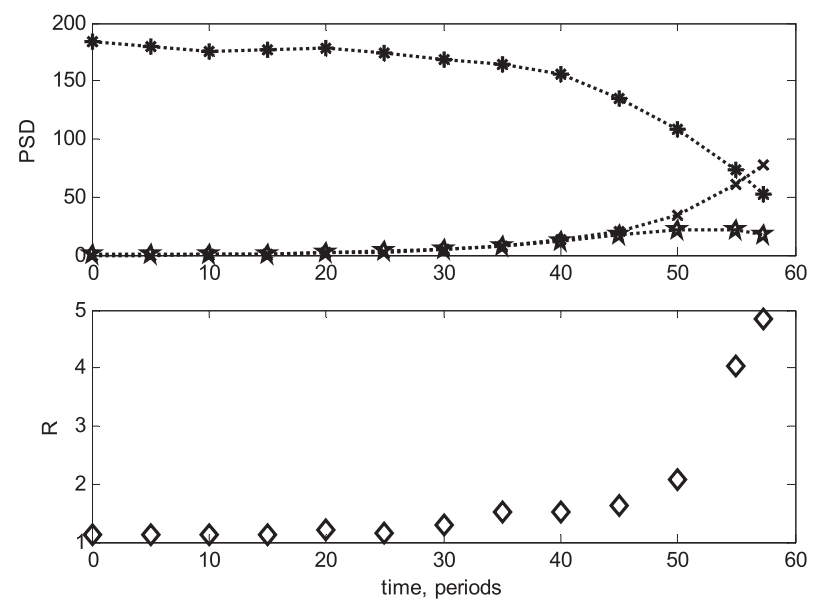

FIG. 1. (top) Power spectral density of the carrier wave $a$ (asterisks), upper sideband $b_{+}$(stars) and lower sideband $b_{-}$(crosses) vs time. (bottom) Modulation depth vs time. Here, $\varepsilon_{1}=0.17, \varepsilon_{1} / \varepsilon_{2}=$ 16.5 , and $U / c=0$.

relative rather than absolute values of these numbers are important.

While propagating, this wave group experiences Benjamin-Feir instability, when new sidebands grow and take energy from the carrier wave. The classical wave system of Benjamin and Feir (Benjamin and Feir 1967) consists of three waves: a carrier wave $a$ and two sidebands $b_{ \pm}$. However, Benjamin-Feir instability is not the primary interest of this study. In our numerical simulations and laboratory experiments, the upper sideband $b_{+}$(sideband with steepness $\varepsilon_{2}$ ) was "seeded" to obtain a broad range of controlled values of modulation depth $R$. In such a case, the second (lower) sideband is not seeded initially but appears from the background noise. When the wave group evolves to breaking, this lower sideband continually grows, and so does the modulation depth $R$. The example of such growth with no wind forcing is shown in Fig. 1.

Sometimes the amplitude of sideband $b_{-}$becomes higher than the carrier wave (i.e., downshifting of the spectral energy occurs). Modulation depth $R$ decreases with the ratio of carrier wave and lower sideband $a / b_{-}$: that is, the higher is $b_{-}$, the larger the modulation depth. In the presence of strong wind forcing, growth of $b_{-}$can be essentially impaired (Fig. 2). In the Fig. 2, the values of ratio $a / b_{-}$at the moment of one period before breaking are plotted versus wind-forcing parameter $U / c$. Here, $U$ is wind speed at the height of half of the wavelength $\lambda / 2$ (in the CS model, all parameters are nondimensional) and $c$ is phase speed of the carrier wave. Figure 2 represents three groups with different values of initial primary wave steepness $\varepsilon_{1}$, whereas the ratio of initial primary steepness and steepness of sideband $\varepsilon_{1} / \varepsilon_{2}$ is the same. Figure 2 


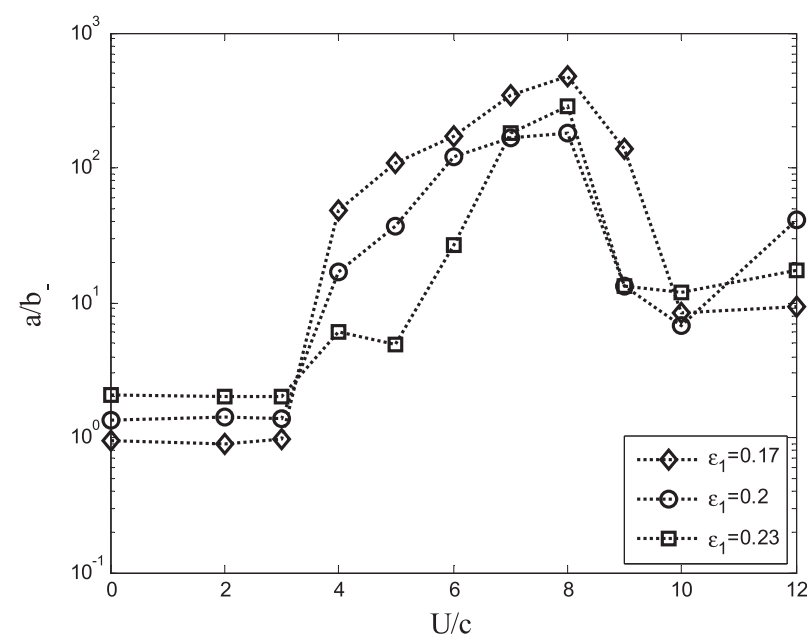

FIG. 2. Ratio of power spectral densities of carrier wave $a$ and lower sideband $b_{-}$at the moment of one period before breaking vs wind forcing for $\varepsilon_{1}=0.17, \varepsilon_{1}=0.2$, and $\varepsilon_{1}=0.23, \varepsilon_{1} / \varepsilon_{2}=60$.

shows that suppression of the lower sideband is especially strong for wind forcing $U / c=6-8$. The broadest range of $a / b_{-}$is for $\varepsilon_{1}=0.17$. It starts from 0.94 (downshifting) for $U / c=0$ and reaches a maximum of 483 for $U / c=8$. The narrowest range is for $\varepsilon_{1}=0.2$. The position of maximum remains the same for all three values of steepness, and that is $U / c=8$. Thus, the position of maximum itself is independent of steepness, but "peak width" decreases with $\varepsilon_{1}$ : for $\varepsilon_{1}=0.17$ a ratio higher than 100 is observed for $U / c=5-9$, whereas for $\varepsilon_{1}=0.23$ it is $U / c=7-8$.

Figure 3 shows how ratio $a / b_{-}$depends on wind forcing for wave groups with the same primary wave steepness $\varepsilon_{1}$ but different $\varepsilon_{1} / \varepsilon_{2}$. In Fig. 3, $a / b_{-}$reaches its maximum for close values $U / c$ in both cases: $U / c=7$ for $\varepsilon_{1} / \varepsilon_{2}=10$ and $U / c=8$ for $\varepsilon_{1} / \varepsilon_{2}=60$. However, values of this maximum noticeably differ: for $\varepsilon_{1} / \varepsilon_{2}=10$ maximum $a / b_{-}=8.7$ and for $\varepsilon_{1} / \varepsilon_{2}=60$ it is 183 . Thus, suppression of lower sideband by wind significantly increases when initial sideband steepness is smaller. This happens because in case of lower initial steepness the lower sideband is suppressed before any Benjamin-Feir instability can develop. As we have mentioned above, modulation depth $R$ depends on $a / b_{-}$; therefore, when $b_{-}$is suppressed by wind, modulation depth is expected to have lower values.

Suppression of Benjamin-Feir instability by the wind for initially monochromatic and modulated wave trains was shown before by a number of researchers. Bliven et al. (1986) found that wind, when imposed on a paddlegenerated "unseeded" (initially monochromatic) wave train, reduces and even (in case of strong wind) suppresses the Benjamin-Feir instability. In their
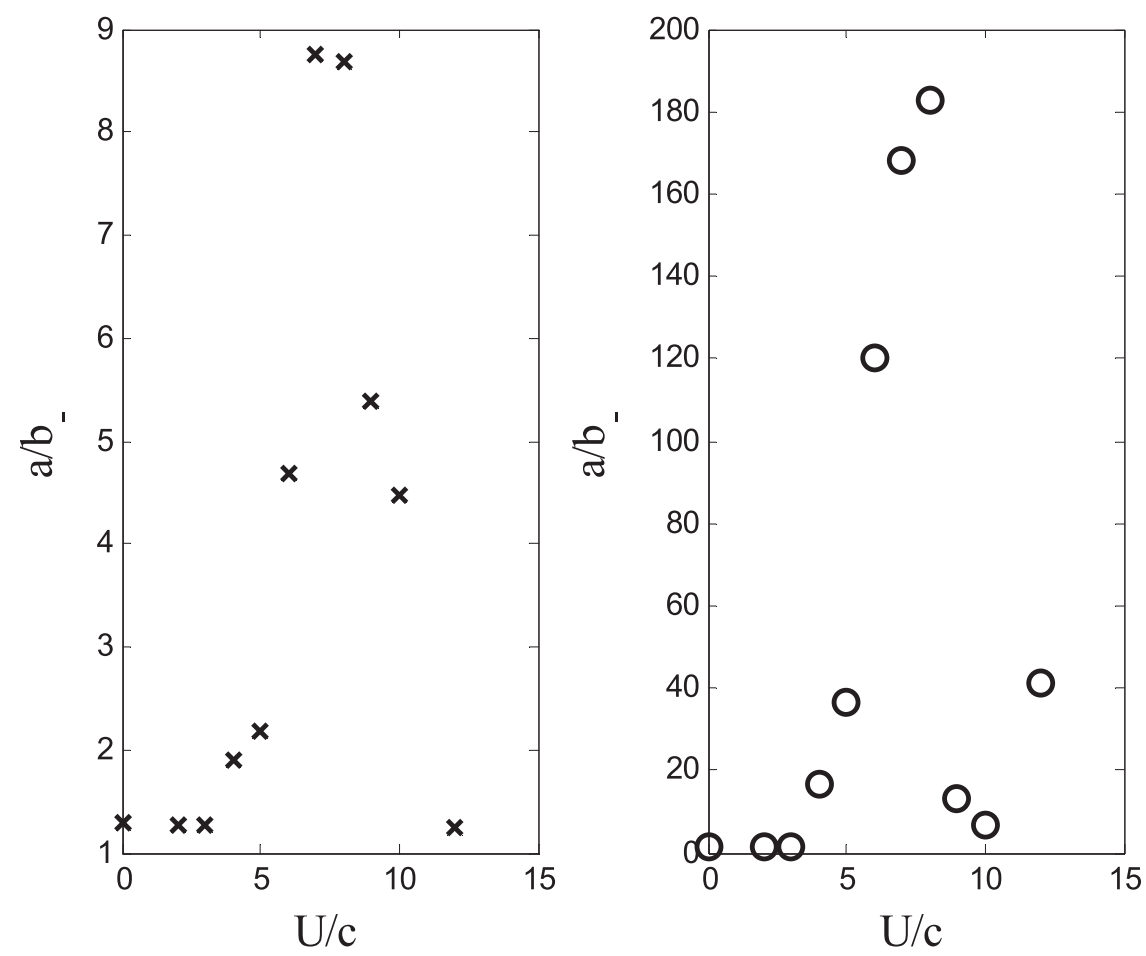

FIG. 3. Ratio of power spectral densities of carrier wave $a$ and lower sideband $b_{-}$vs wind forcing for $\varepsilon_{1}=0.2$, (left) $\varepsilon_{1} / \varepsilon_{2}=10$ and (right) $\varepsilon_{1} / \varepsilon_{2}=60$. 
experiments, sideband magnitude, growth rate, and low-frequency perturbation components associated with the instability mechanism were reduced when the wind speed increased. The results of Bliven et al. (1986) were qualitatively confirmed by numerical simulations of Trulsen and Dysthe (1992). They used a modification of nonlinear Schrödinger equation, to which they added two source terms: action of wind and wave breaking. Simulations of Trulsen and Dysthe showed that for low winds and breaking the evolution of wave trains was quantitatively the same as for the situation with breaking but without winds; that is, for low winds they observed downshifting of the dominant frequency and the Benjamin-Feir instability. For stronger winds, the situation was different: modulational instability existed but was significantly slowed down and delayed in time.

Another thorough investigation on the topic was carried out by Waseda and Tulin (1999). They conducted laboratory experiments with both initially monochromatic wave trains and wave groups with two seeded sidebands. In the paper, they report that the growth rates of the sidebands were reduced for weak wind forcing and enhanced for strong wind forcing. This seems to contradict the previous results obtained by Bliven et al. (1986). However, Bliven et al. did not estimate growth rate but reported a suppression of the sideband energy.

One of the most recent works considering the influence of wind on Benjamin-Feir instability is that by Kharif et al. (2010). Following Segur et al. (2005), who proved numerically and experimentally that viscous dissipation can bound the growth of perturbations "before nonlinearity comes into play," Kharif et al. (2010) numerically investigated a wave system with both dissipation and wind. They found that, in the presence of both dissipation and wind, the instability in a weakly nonlinear modulated wave train occurs when friction velocity is higher than some critical velocity. Critical velocity defines the minimum friction velocity induced by the wind to amplify a wave train with a certain carrier wave frequency (critical velocity increases with wavelength or decreases with frequency of the carrier wave). Kharif et al. (2010) note that "in the presence of wind and dissipation, the unstable domain shrinks for lowfrequency regime: this means that young waves are more sensitive to Benjamin-Feir instability than old waves." The research of Kharif et al. (2010) was continued by Touboul and Kharif (2010), where they used a twodimensional fully nonlinear model, and the marginal stability curve they obtained coincided with the curve obtained with the weakly nonlinear version of Kharif et al. (2010).
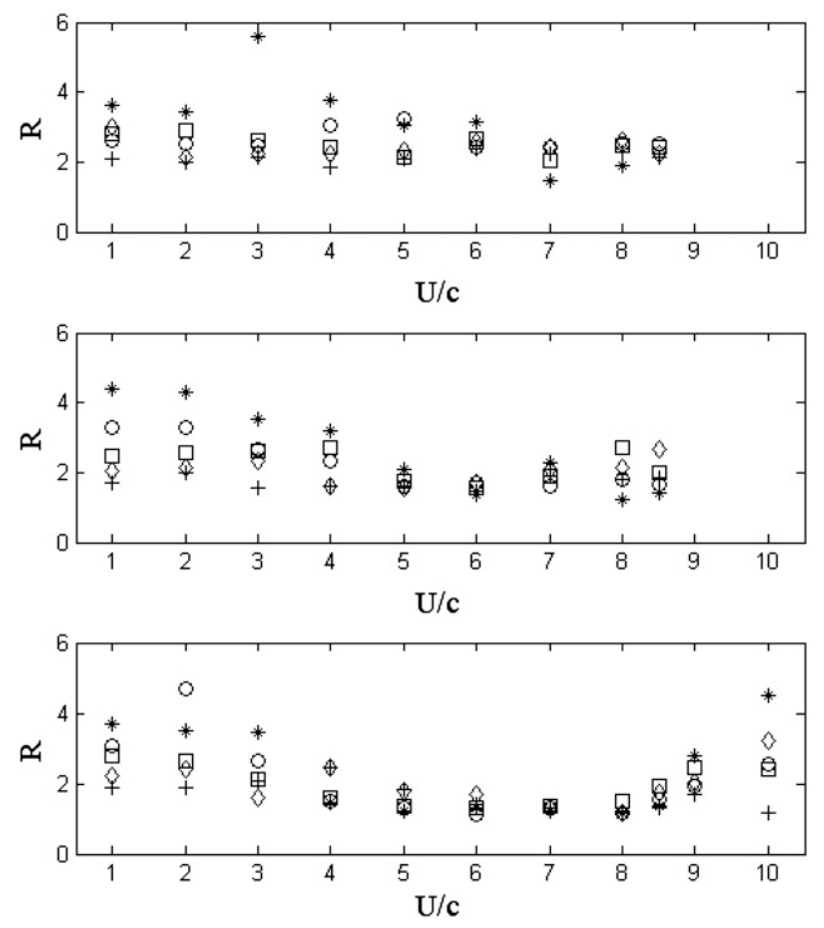

FIG. 4. Modulation depth $R$ as a function of wind forcing for $\varepsilon_{1}=$ 0.17 (asterisks), $\varepsilon_{1}=0.19$ (circles), $\varepsilon_{1}=0.21$ (squares), $\varepsilon_{1}=0.23$ (diamonds), and $\varepsilon_{1}=0.25$ (pluses): (top) $\varepsilon_{1} / \varepsilon_{2}=5$, (middle) $\varepsilon_{1} / \varepsilon_{2}=$ 10 , and (bottom) $\varepsilon_{1} / \varepsilon_{2}=40$.

\section{Lifetime and modulation depth as a function of wind forcing}

Above it was shown that, as a consequence of lower sideband suppression, decrease of modulation depth (as measured at the moment of one period before breaking) can be expected when wind forcing is increasing up to $U / c=6-8$. Figure 4 represents dependence of modulation depth $R$ on wind forcing $U / c$ for different values of initial primary wave steepness. The difference between the three panels in Fig. 4 is in ratio $\varepsilon_{1} / \varepsilon_{2}$. Comparing the three cases, one can see that modulation depth is being reduced by wind forcing more noticeably for higher ratios of primary and secondary wave steepness $\varepsilon_{1} / \varepsilon_{2}$. In the top panel of Fig. $4, \varepsilon_{1} / \varepsilon_{2}=5$ and the decrease of modulation depth is insignificant, whereas for $\varepsilon_{1} / \varepsilon_{2}=10$ (Fig. 4, middle) $R$ decreases $1.5-2$ times for low values of primary wave steepness at $U / c=6$ (compared to values at $U / c=1)$ and then again increases. Minimum of modulation depth and further increase is especially evident for $\varepsilon_{1} / \varepsilon_{2}=40$ : that is, for smaller initial sideband steepness. An interesting fact is that lifetime repeats this trend, though approximately, and also shows a minimum and then further increase (Fig. 5). The effect of increasing lifetime for extreme winds is almost absent for $\varepsilon_{1} / \varepsilon_{2}=5$ (Fig. 5 , top), hardly noticeable for $\varepsilon_{1} / \varepsilon_{2}=10$ 

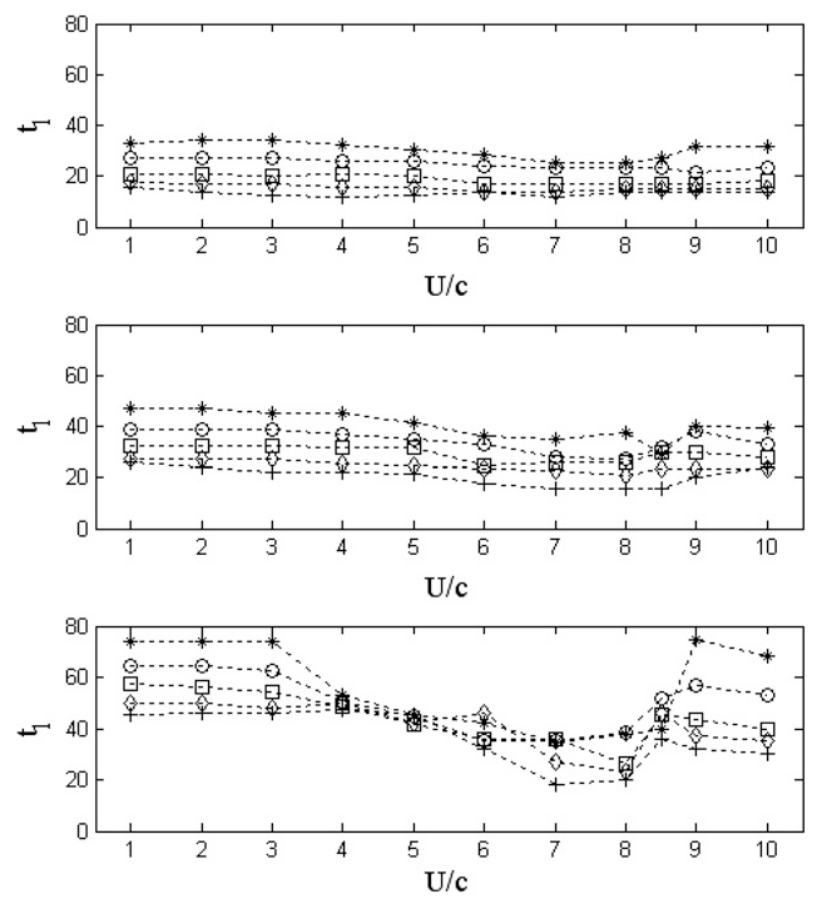

FIG. 5. Lifetime $t_{l}$ in periods as a function of wind forcing for $\varepsilon_{1}=$ 0.17 (asterisks), $\varepsilon_{1}=0.19$ (circles), $\varepsilon_{1}=0.21$ (squares), $\varepsilon_{1}=0.23$ (diamonds), and $\varepsilon_{1}=0.25$ (pluses): (top) $\varepsilon_{1} / \varepsilon_{2}=5$, (middle) $\varepsilon_{1} / \varepsilon_{2}=$ 10 , and (bottom) $\varepsilon_{1} / \varepsilon_{2}=40$.

(Fig. 5, middle), but strongly pronounced for a higher $\varepsilon_{1} / \varepsilon_{2}=40$ (Fig. 5, bottom). The reduction of lifetime for wind forcing $U / c=6-8$ means that the probability of breaking grows, whereas modulation depth is close to 1 (i.e., no modulation). This, in its turn, means that for such wind forcing modulational instability is no longer a reason for breaking.

Galchenko et al. (2010) showed that both lifetime (distance to breaking) and modulation depth decrease with primary wave steepness. In the presence of wind, lifetime also diminishes with primary wave steepness for any value of wind forcing, which means that for the same wind forcing probability of breaking is higher for initially steeper waves (Fig. 6). With the modulation depth, the situation is more complicated. Whether modulation depth depends on $\varepsilon_{1}$ or not, it is defined by wind forcing. For low to moderate wind forcing, $R$ depends on $\varepsilon_{1}$. For strong wind forcing $(U / c>5)$, modulation depth does not depend on primary wave steepness any more (Fig. 7). Apparently, such wind forcing makes the waves grow to the limiting steepness before the modulational instability can take its course. In the case of $U / c<5$, the dependence of modulation depth on primary wave steepness $\varepsilon_{1}$ can be different: it can either decrease, when $\varepsilon_{1} / \varepsilon_{2}$ is high, or increase, when $\varepsilon_{1} / \varepsilon_{2}$ is low. This dependence repeats the dependence of ratio $a / b_{-}$on $\varepsilon_{1}$ (Figs. 8, 9).

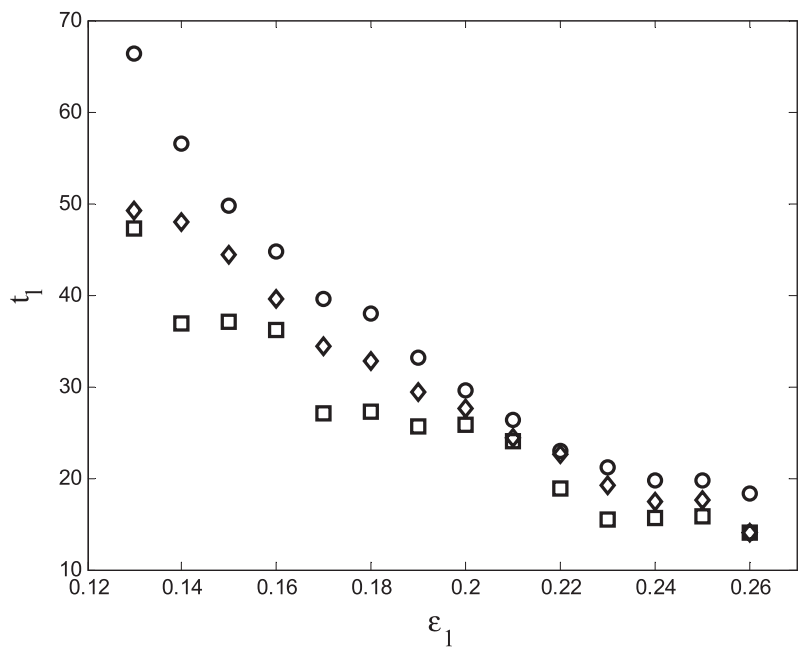

FIG. 6. Lifetime $t_{l}$ vs initial primary wave steepness $\varepsilon_{1}$ for three values of wind forcing, $U / c=2$ (circles), $U / c=5$ (diamonds), and $U / c=8$ (squares): $\varepsilon_{1} / \varepsilon_{2}=7$.

For lower ratio $\varepsilon_{1} / \varepsilon_{2}$ modulation depth decreases with $\varepsilon_{1}$ (e.g., $\varepsilon_{1} / \varepsilon_{2}=7$ in Fig. 8 ), but for higher $\varepsilon_{1} / \varepsilon_{2}$ it grows (e.g., $\varepsilon_{1} / \varepsilon_{2}=60$ in Fig. 8 ).

Here, it is important to note that ratio of steepnesses $\varepsilon_{1} / \varepsilon_{2}$ is not the only possible parameter for describing the dynamics of nonlinear wave groups. Benjamin-Feir instability is controlled not just by nonlinearity (the decrease of $\varepsilon_{1} / \varepsilon_{2}$ corresponds to the decrease of nonlinearity) but also by dispersion: that is, bandwidth in the system. In our previous and current laboratory experiments on the breaking severity, we concentrated on controlling modulation depth immediately before the breaking, which was achieved by manipulating $\varepsilon_{1} / \varepsilon_{2}$. In

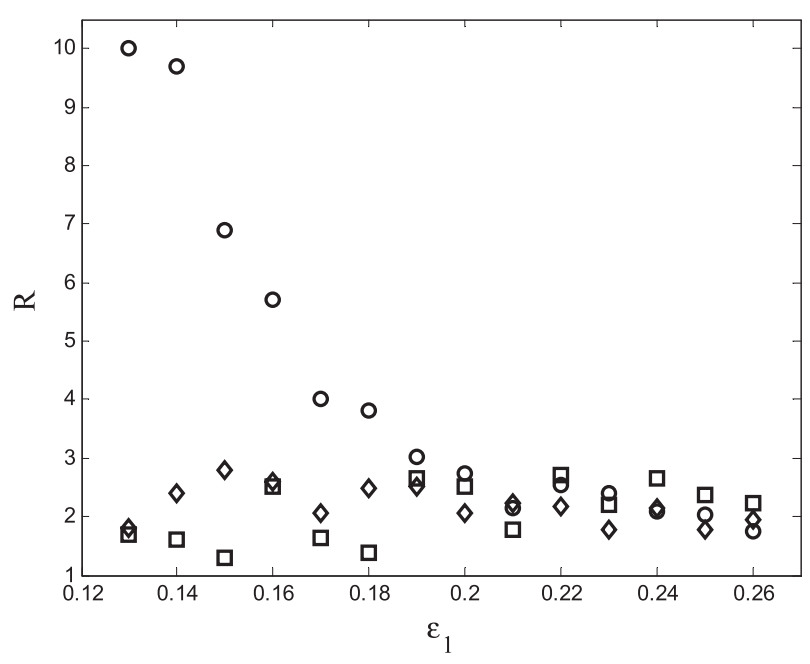

FIG. 7. Modulation depth $R$ vs initial primary wave steepness $\varepsilon_{1}$ for three values of wind forcing, $U / c=2$ (circles), $U / c=5$ (diamonds), and $U / c=8$ (squares): $\varepsilon_{1} / \varepsilon_{2}=7$. 


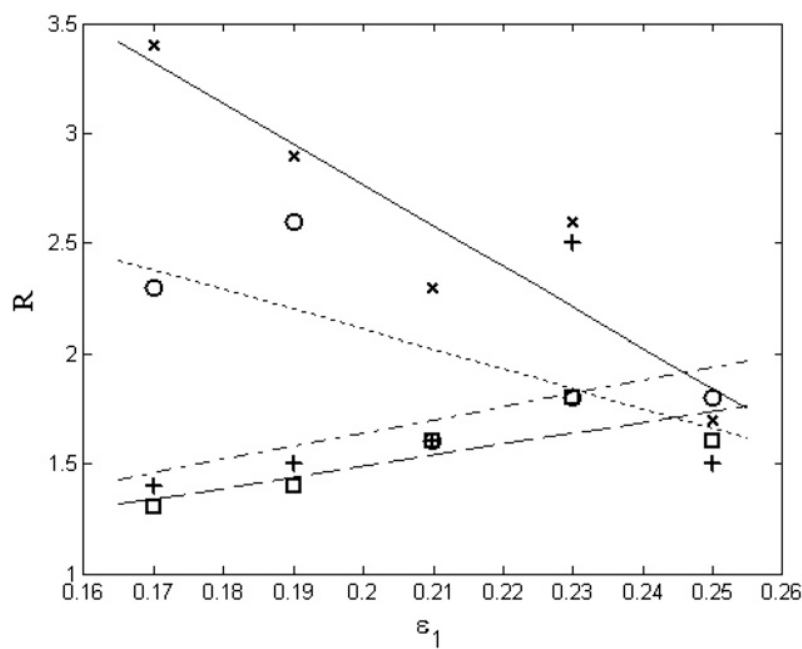

FIG. 8. Modulation depth as a function of initial primary wave steepness $\varepsilon_{1}: U / c=4$. Here, $\varepsilon_{1} / \varepsilon_{2}=7$ (crosses), $\varepsilon_{1} / \varepsilon_{2}=20$ (circles), $\varepsilon_{1} / \varepsilon_{2}=40$ (pluses), and $\varepsilon_{1} / \varepsilon_{2}=60$ (squares). Straight lines are linear fits: $\varepsilon_{1} / \varepsilon_{2}=7$ (solid), $\varepsilon_{1} / \varepsilon_{2}=20$ (dotted), $\varepsilon_{1} / \varepsilon_{2}=40$ (dasheddotted), and $\varepsilon_{1} / \varepsilon_{2}=60$ (dashed).

the present research, initial bandwidth (also initial number of waves in a group) was not varied. In the numerical simulations, we have performed an investigation on response of modulation depth to variations of bandwidth. Such dependence without wind is shown in Fig. 10. The most obvious changes in modulation depth with bandwidth occur for smaller initial steepness. Figure 11 shows dependence (or, to be more precise, no certain dependence) of $R$ on bandwidth for $U / c=2$ and $U / \mathrm{c}=4$. Maximum modulation depth for $U / c=2$ is $R=3$ and for $U / C=4$ is $R=3.4$; both maximums are

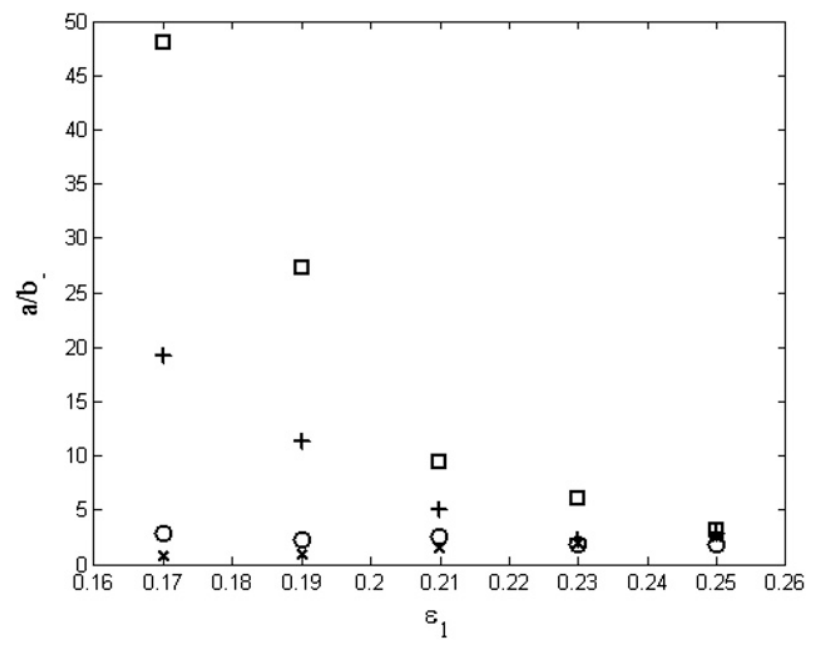

FIG. 9. Ratio of amplitudes of carrier wave $a$ and lower sideband

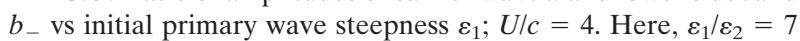
(crosses), $\varepsilon_{1} / \varepsilon_{2}=20$ (circles), $\varepsilon_{1} / \varepsilon_{2}=40$ (pluses), and $\varepsilon_{1} / \varepsilon_{2}=60$ (squares).
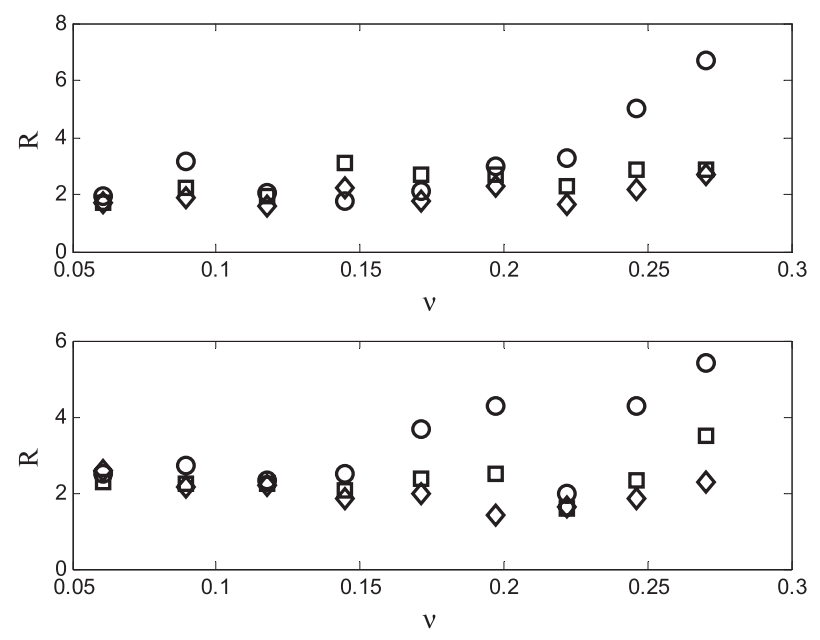

FIG. 10. Modulation depth vs bandwidth for (top) $\varepsilon_{1} / \varepsilon_{2}=40$ and (bottom) $\varepsilon_{1} / \varepsilon_{2}=7: \varepsilon_{1}=0.18$ (circles), $\varepsilon_{1}=0.21$ (squares), and $\varepsilon_{1}=$ 0.24 (diamonds).

reached for $\nu=0.197$. Minimums are $R=2.3$ and $R=$ 1.8 , respectively, so the difference between maximum and minimum for the range of bandwidths shown in Fig. 11 is 0.7 for $U / c=2$ and 1.8 for $U / c=4$. In Fig. 12, one can see dependence of modulation depth on ratio $\varepsilon_{1} / \varepsilon_{2}$ for different values of bandwidth for two wind forcings. For smaller wind forcing, $R$ is independent of $\varepsilon_{1} / \varepsilon_{2}$; for higher wind forcing, $U / c=4$, it generally decreases for all values of bandwidth. We investigated influence of wind on this dependence for different values of initial steepness $\varepsilon_{1}$ and the same bandwidth $\nu=0.145$ and found that without wind, for small winds, and for extreme winds such dependence does not exist, whereas for high winds modulation depth decreases with $\varepsilon_{1} / \varepsilon_{2}$ (Fig. 13), just as it does for other bandwidths in Fig. 12.

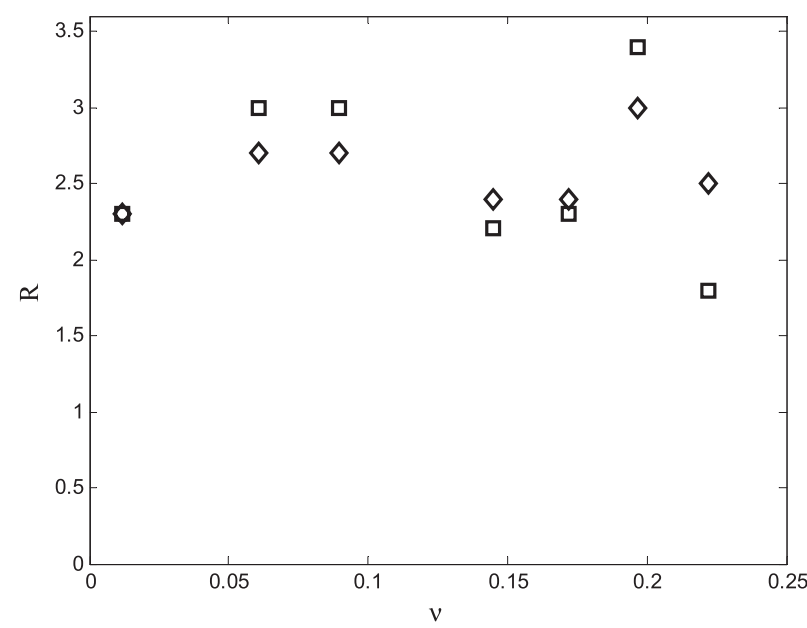

FIG. 11. Modulation depth vs bandwidth for $U / c=2$ (diamonds) and $U / c=4$ (squares): $\varepsilon_{1}=0.21$ and $\varepsilon_{1} / \varepsilon_{2}=7$. 

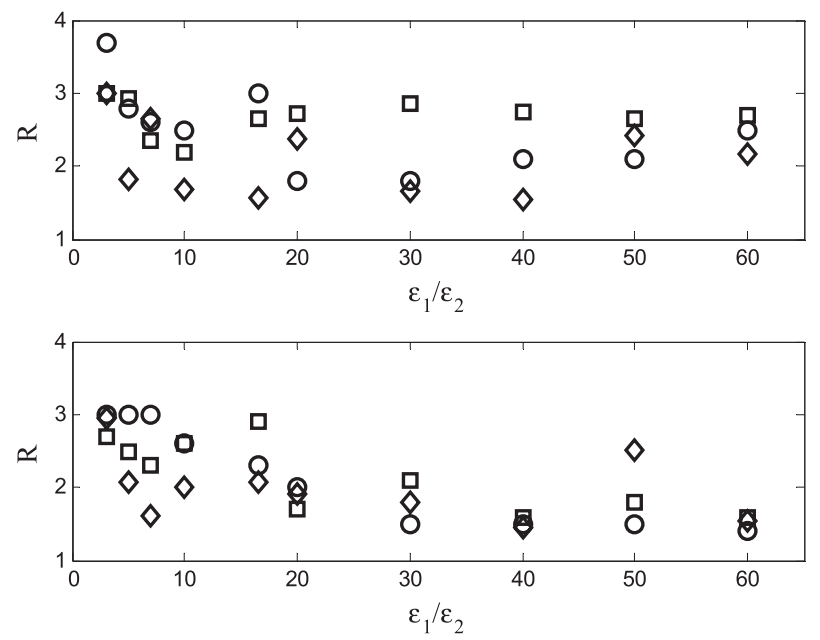

FIG. 12. Modulation depth vs ratio of steepnesses $\varepsilon_{1} / \varepsilon_{2}$ : (top) $U / c=2$ and (bottom) $U / c=4$. Here, $\nu=0.0606$ (circles), $\nu=0.145$ (squares), and $\nu=0.222$ (diamonds).

\section{Wind and breaking severity}

Galchenko et al. (2010) showed that in the absence of wind severity of breaking grows with modulation depth. Also, it was found that probability of breaking for wave groups with $R<2.2$ is very low. Here, numerical simulations (section 3 ) show that wind forcing causes the decrease of modulation depth and at the same time significantly increases the probability of breaking for wave groups with $1<R<2.2$ : under strong wind waves break even in groups with $R$ close to 1 . This means that with wind forcing severity of breaking is expected to decrease.

This expectation was confirmed experimentally. The laboratory experiment with wind forcing was conducted in the Air-Sea Interaction Saltwater Tank (ASIST) at the Rosenstiel School of Marine and Atmospheric Science, University of Miami. The sketch of the experimental setup is shown in Fig. 14. Wave groups with wind forcing were produced by a digitally controlled mechanical wave generator and a wind generator. Laser elevation gauges with cameras measured surface elevations at a rate of $250 \mathrm{~Hz}$ with a vertical pixel resolution of $0.2 \mathrm{~mm}$ as described in Savelyev et al. (2011). Camera 1 recorded initial conditions, and breaking occurred between cameras 2 and 3 .

Figure 15 shows the dependence of modulation depth on primary wave steepness for two values of wind obtained numerically and experimentally. In Fig. 15, numerical values for modulation depth slightly overestimate experimental values for the case of smaller wind forcing, $U / c=2$. Quasi two dimensionality of the experimental conditions is most probably responsible for this overestimation.
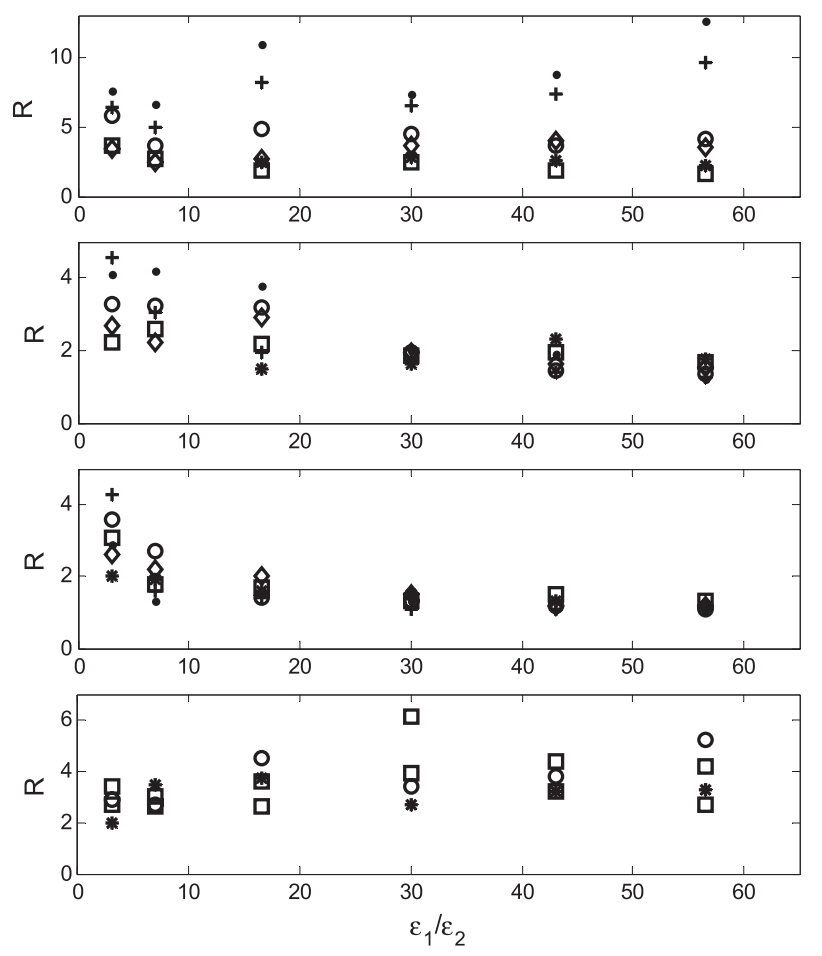

FIG. 13. Modulation depth as a function of ratio $\varepsilon_{1} / \varepsilon_{2}$ for $\varepsilon_{1}=$ 0.15 (dots), $\varepsilon_{1}=0.17$ (pluses), $\varepsilon_{1}=0.19$ (circles), $\varepsilon_{1}=0.21$ (diamonds), $\varepsilon_{1}=0.23$ (squares), and $\varepsilon_{1}=0.25$ (asterisks). (top)(bottom) No wind $U / c=0, U / c=4, U / c=8$, and $U / c=11$.

Breaking severity coefficient was defined as follows:

$$
S=\frac{E_{\mathrm{bb}}-E_{\mathrm{ab}}}{E_{\mathrm{bb}}},
$$

where $E_{\mathrm{bb}}$ is the wave energy before breaking and $E_{\mathrm{ab}}$ is the energy after breaking. Thus, the severity coefficient $S$ is a nondimensional parameter. By the energy, we mean potential energy of the wave group,

$$
E=\int_{0}^{T} \eta^{2}(t) d t
$$

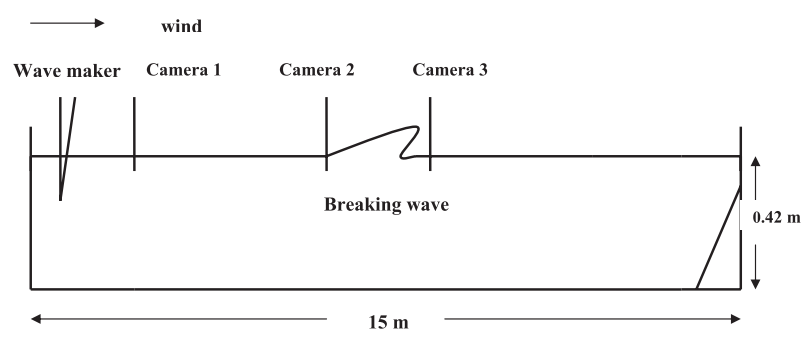

FIG. 14. Experimental setup scheme. Proportions are arbitrary. The distance from the wavemaker to cameras 2 and 3 is 9.15 and $10.65 \mathrm{~m}$, respectively. 

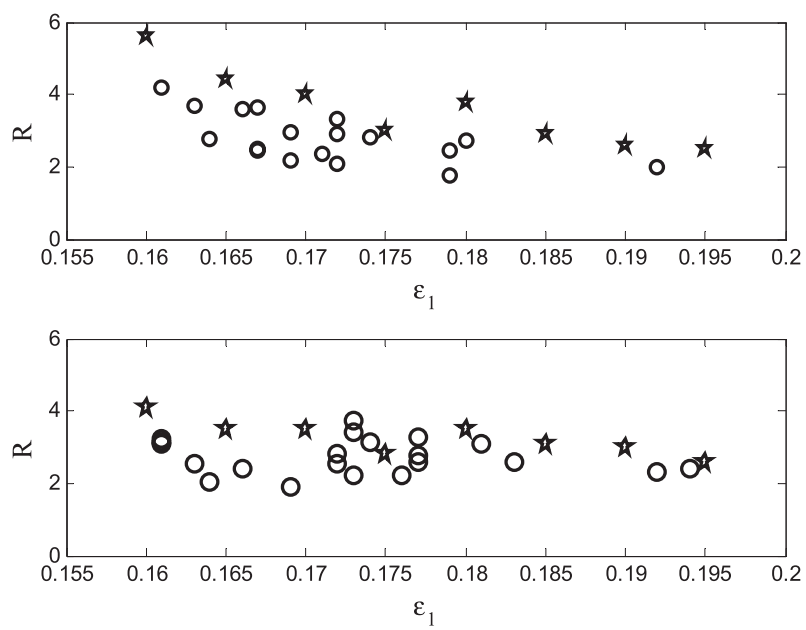

FIG. 15. Modulation depth vs primary wave steepness for (top) $U / c=2$ and (bottom) $U / c=3.8$. Numerical data (stars) and experimental data (circles) are shown; $\varepsilon_{1} / \varepsilon_{2} \approx 7$.

where $\eta(t)$ is time series of surface elevations and $T$ is the period of the whole group.

Figure 16 shows dependence of severity coefficient $S$ on wind forcing U/c. In Fig. 16 (bottom), data obtained for a certain wind forcing are averaged. Even though the scatter of data is significant (Fig. 16, top), one can notice that severity coefficient decreases with wind forcing. The data in Fig. 16 are not sorted out by initial steepnesses or any other initial conditions. Sorting by the modulation depth, where ranges of which are shown with different markers in Fig. 16 (top), does not appear to improve the scatter. Overall severity, however, does decrease with wind, which is consistent with laboratory observations of Babanin et al. (2010).

Figure 17 shows how severity coefficient grows with modulation depth for two values of wind forcing. One can notice that, for weaker wind forcing of $U / c=2\left(U_{10}=\right.$ $\left.5 \mathrm{~m} \mathrm{~s}^{-1}\right)$, values of severity coefficients are on average somewhat higher than for stronger wind forcing $U / c=$ $3.8\left(U_{10}=10 \mathrm{~m} \mathrm{~s}^{-1}\right)$. This could be expected from the results presented in Fig. 16: severity decreases with the wind. Also, for stronger wind forcing $U / c=3.8$ the scatter is more noticeable than for $U / c=2$. The explanation of this fact follows from the influence of wind on Benjamin-Feir instability discussed in the previous sections: for stronger winds, instability plays a less important role. It can be expected that further increase of wind (i.e., extreme wind conditions) would weaken and may even cancel the dependence $S(R)$; however, this is subject to future studies.

Comparing the results of Galchenko et al. (2010) (breaking severity versus wind forcing in the absence of wind) with results of Fig. 17, we can see that the range of severity coefficients in the absence of wind is much
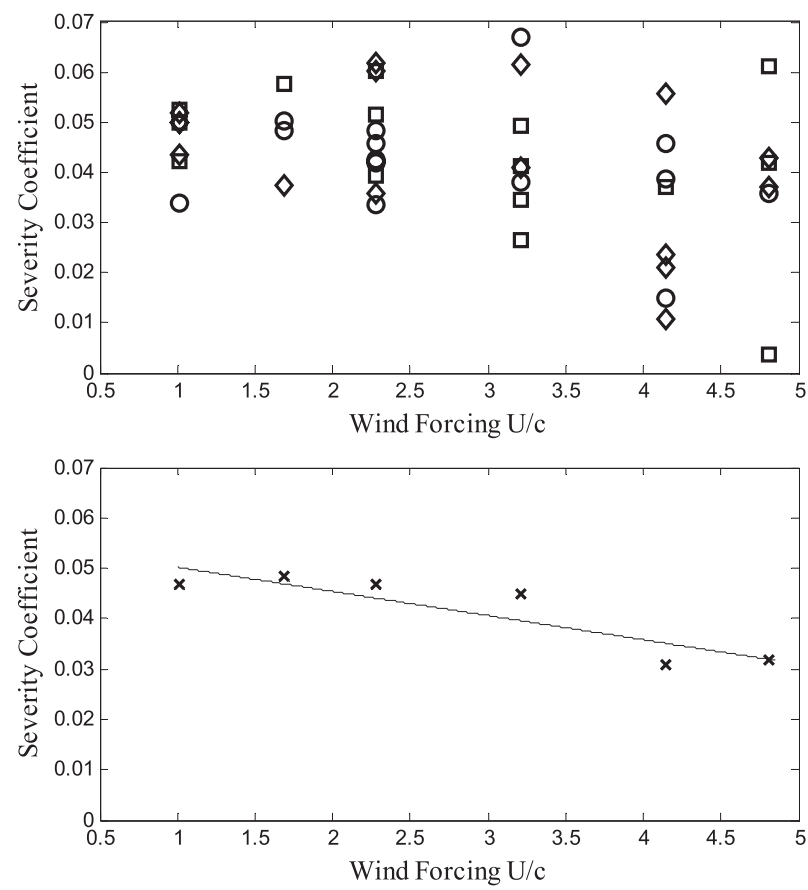

FIG. 16. Breaking severity as a function of wind forcing. (top) $R<2.3$ (circles), $2.3<R<2.7$ (squares), and $R>2.7$ (diamonds). (bottom) As in (top), but averaged for every value of wind forcing: laboratory observations.

higher than in the cases with wind (Fig. 18). Maximum energy loss observed in the experiment of Galchenko et al. (2010) without wind forcing (asterisks in Fig. 18) was $36 \%$, and for the experiment with wind it is $6 \%$. Another difference between the wind and no-wind case is that there are nonzero values of energy loss for $R$ close to 1 (i.e., for groups with practically no modulation).

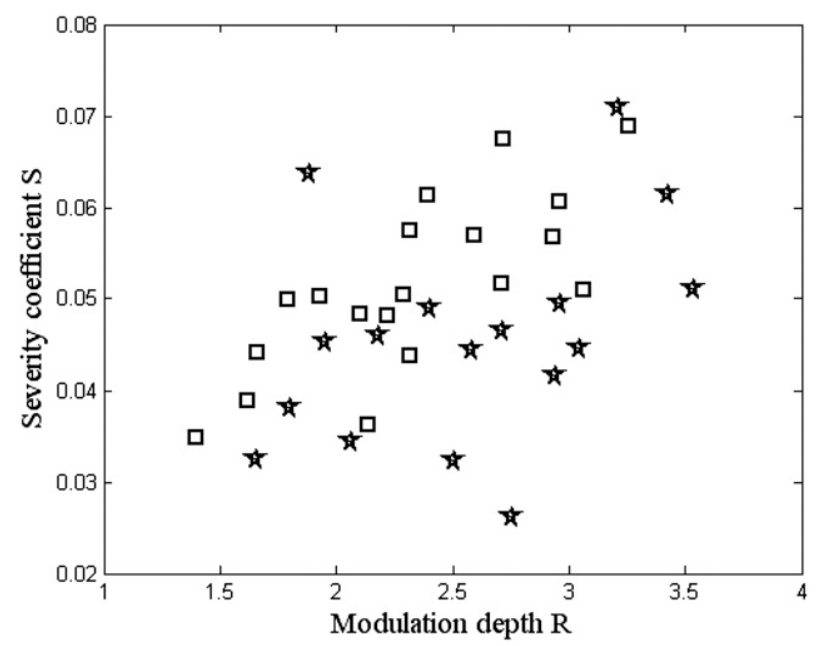

FIG. 17. Breaking severity vs modulation depth in the presence of wind forcing $U / c=2$ (squares) and $U / c=3.8$ (stars): laboratory observations. 


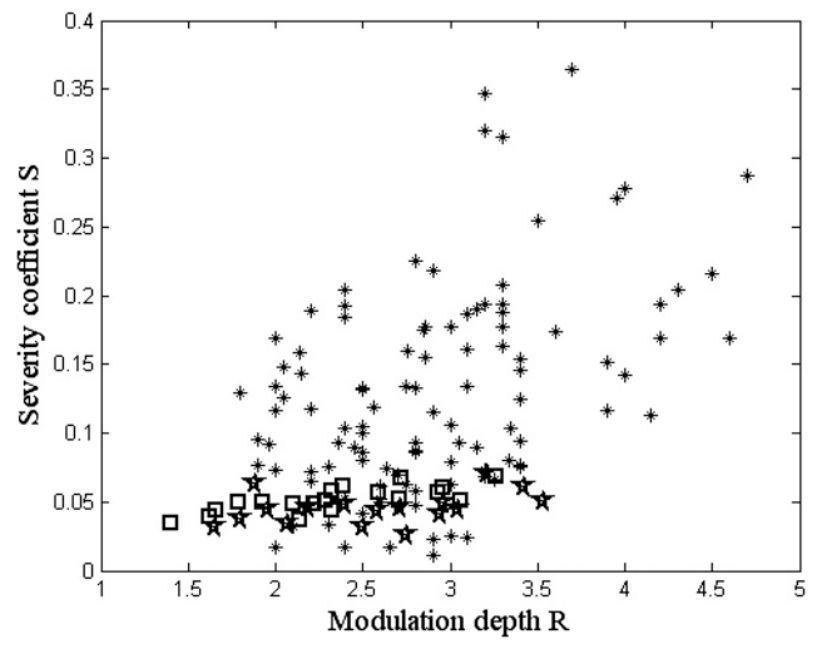

FIG. 18. Breaking severity vs modulation depth for $U / c=0$ (asterisks), $U / c=2$ (squares), and $U / c=3.8$ (stars): laboratory observations.

This means that such waves break because of reasons other than Benjamin-Feir instability; for example, they may grow to the limiting steepness because of the direct wind forcing before this instability takes an effect.

\section{Discussion and conclusions}

Progression of one-dimensional nonlinear wave groups to breaking in the presence of wind forcing was studied by means of a fully nonlinear numerical model and experimentally. It was shown that for certain values of wind forcing, Benjamin-Feir instability is significantly suppressed and modulation depth decreases, whereas breaking probability increases. An opposite effect is, however, observed for extreme wind forcing.

The probability of breaking for wave groups with $1<$ $R<2.2$, being very low without wind forcing, grows sharply in the presence of wind. This means that in the presence of such wind forcing there are reasons for breaking other than Benjamin-Feir instability.

The range of breaking severity coefficients with wind forcing is much narrower than without wind forcing. Wind increases breaking probability but decreases the severity of a single breaker.

An approximate estimation of dependence of wave energy dissipation on modulation depth can be made using the numerical and experimental data described above. Figure 19 demonstrates numerically obtained inverse lifetime (or breaking probability $1 / t_{l}$ ); severity coefficient $S$, averaged from experimental data for the corresponding values of $R$; and dissipation defined as $D=$ $\left(1 / t_{l}\right) S$ as functions of modulation depth $R$ for two values of wind forcing. In Fig. 19, for $U / c=2$ dissipation
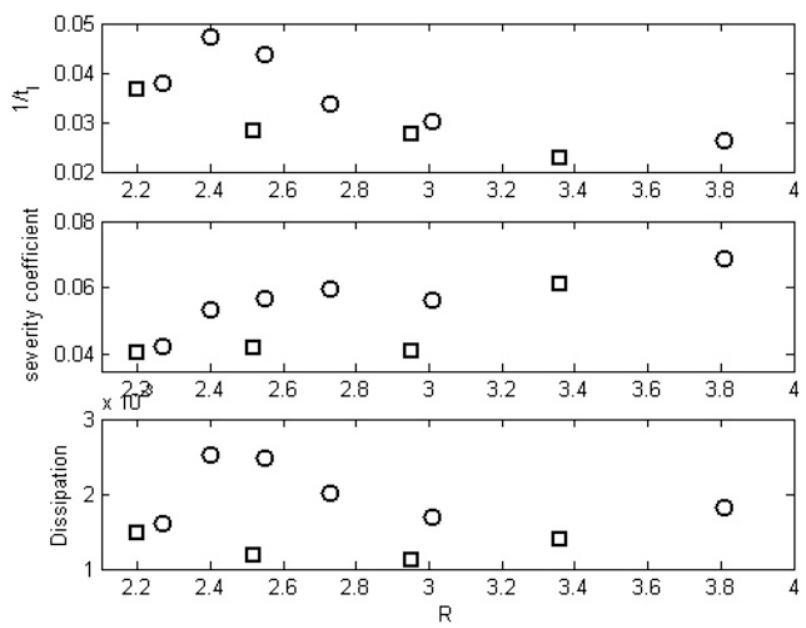

FIG. 19. (top) Breaking probability, (middle) severity coefficient, and (bottom) dissipation vs modulation depth in the presence of wind: $U / c=2$ (circles) and $U / C=3.8$ (squares).

decreases and reaches saturation at $R>2.8$. At the stronger wind forcing of $U / c=3.8$, the dissipation is rather constant and on average smaller than that for $U / c=2$.

Acknowledgments. We thank Dahai Jeong for her assistance with the laboratory experiment. A. V. Babanin and I. R. Young gratefully acknowledge financial support of the Australian Research Council and Woodside Energy Ltd through the Linkage Grant LP0883888 and of the Australian Research Council through the Discovery Grants DP1093349 and DP1093517.

\section{APPENDIX A}

\section{Maximum and Minimum Energy Loss for Different Values of Wind}

Shown in this appendix are the severity coefficients (Table A1).

\section{APPENDIX B}

\section{Fits and Their Accuracy}

Shown in this appendix are the correlation coefficients for Figs. 8, 11, and 13 (Tables B1-B3, respectively).

TABLE A1. Severity coefficients.

\begin{tabular}{lcc}
\hline \hline$U / c$ & $S_{\min }(\%)$ & $S_{\max }(\%)$ \\
\hline 0 & 1.1 & 36 \\
2 & 3.5 & 6.9 \\
3.8 & 2.6 & 7.1 \\
\hline
\end{tabular}


TABLE B1. Correlation coefficients for Fig. 8.

\begin{tabular}{clc}
\hline \hline$\varepsilon_{1} / \varepsilon_{2}$ & \multicolumn{1}{c}{ Linear fit } & Correlation coef \\
\hline 7 & $y=-18.5 x+6.5$ & 0.917 \\
20 & $y=-9 x+3.91$ & 0.6862 \\
40 & $y=6 x+0.44$ & 0.4191 \\
60 & $y=5 x+0.49$ & 0.8111 \\
\hline
\end{tabular}

TABLE B2. Correlation coefficient for Fig. 11

\begin{tabular}{cc}
\hline \hline Linear fit & Correlation coef \\
\hline$y=-0.0048 x+0.055$ & 0.8894
\end{tabular}

TABLE B3. Correlation coefficients for Fig. 13.

\begin{tabular}{ccc}
\hline \hline$U / c$ & Linear fit & Correlation coef \\
\hline 0 & $\mathrm{y}=0.161 \mathrm{x}-0.35$ & 0.4378 \\
2 & $\mathrm{y}=0.19 \mathrm{x}+0.007$ & 0.7564 \\
\hline
\end{tabular}

\section{REFERENCES}

Alber, I. E., 1978: The effects of randomness on the stability of twodimensional wavetrains. Proc. Roy. Soc. London, A363, 525-546.

Babanin, A. V., 2009: Breaking of ocean surface waves. Acta Phys. Slovaca, 59, 305-535.

_ I. R. Young, and M. L. Banner, 2001: Breaking probabilities for dominant surface waves on water of finite constant depth. J. Geophys. Res., 106C, 11 659-11 676.

_ M. L. Banner, I. R. Young, and M. A. Donelan, 2007a: Wavefollower field measurements of the wind-input spectral function. Part III: Parameterization of the wind-input enhancement due to wave breaking. J. Phys. Oceanogr., 37, 2764-2775.

- D. Chalikov, I. Young, and I. Savelyev, 2007b: Predicting the breaking onset of surface water waves. Geophys. Res. Lett., 34, L07605, doi:10.1029/2006GL029135.

$\longrightarrow,-,-$, and,- 2010 : Numerical and laboratory investigation of breaking of steep two-dimensional waves in deep water. J. Fluid Mech., 644, 433-463.

— - T. Waseda, T. Kinoshita, and A. Toffoli, 2011a: Wave breaking in directional fields. Phys. Oceanogr., 41, 145-156.

$\longrightarrow$, - I. Shugan, and H.-H. Hwung, 2011b: Modulational instability in directional wave fields and extreme wave events. Proc. 30th Int. Conf. on Ocean, Offshore and Arctic Engineering (OMAE2011), Rotterdam, Netherlands, American Society of Mechanical Engineers, OMAE2011-49540.

Banner, M. L., A. V. Babanin, and I. R. Young, 2000: Breaking probability for dominant waves on the sea surface. J. Phys. Oceanogr., 30, 3145-3160.

Benjamin, T. B., and J. E. Feir, 1967: The disintegration of wave trains in deep water. Part 1. Theory. J. Fluid Mech., 27, 417430.

Bliven, L., N. Huang, and S. Long, 1986: Experimental study of the influence of wind on Benjamin-Feir sideband instability. J. Fluid Mech., 162, 237-260.

Cavaleri, L., and Coauthors, 2007: Wave modeling-The state of the art. Prog. Oceanogr., 75, 603-674.
Chalikov, D., and D. Sheinin, 2005: Modelling extreme waves based on equations of potential flow with a free surface. Comput. Phys., 210, 247-273.

_ , and S. Rainchik, 2011: Coupled numerical modelling of wind and waves and a theory of wave boundary layer. Bound.-Layer Meteor., 138, 1-41, doi:10.1007/s10546-010-9543-7.

Donelan, M. A., A. V. Babanin, I. R. Young, M. L. Banner, and C. McCormick, 2005: Wave-follower field measurements of the wind-input spectral function. Part I: Measurements and calibrations. J. Atmos. Oceanic Technol., 22, 799-813.

,,,--- and,- 2006 : Wave-follower measurements of the wind-input spectral function. Part II: Parameterization of the wind input. J. Phys. Oceanogr., 36, 1672-1688.

Galchenko, A., A. V. Babanin, D. Chalikov, I. R. Young, and T. W. Hsu, 2010: Modulational instabilities and breaking strength for deep-water wave groups. J. Phys. Oceanogr., 40, 2313-2324.

Guan, C., W. Hu, J. Sun, and R. Li, 2007: The whitecap coverage model from breaking dissipation parametrizations of wind waves. J. Geophys. Res., 112, C05031, doi:10.1029/2006JC003714.

Hwang, P. A., D. Xu, and J. Wu, 1989: Breaking of wind-generated waves: Breaking and characteristics. J. Fluid Mech., 202, 177-200.

Kharif, C., R. A. Kraenkel, M. A. Manna, and R. Thomas, 2010: The modulational instability in deep water under the action of wind and dissipation. J. Fluid Mech., 664, 138-149.

Manasseh, R., A. V. Babanin, C. Forbes, K. Rickards, I. Bobevski, and A. Ooi, 2006: Passive acoustic determination of wavebreaking events and their severity across the spectrum. J. Atmos. Oceanic Technol., 23, 599-618.

McLean, J. W., 1982a: Instabilities of finite-amplitude gravity waves on water of finite depth. J. Fluid Mech., 114, 331-341.

_ 1982b: Instabilities of finite-amplitude water waves. J. Fluid Mech., 114, 315-330.

Monahan, E. C., 1971: Oceanic whitecaps. J. Phys. Oceanogr., 1, 139-144.

Mori, N., M. Onorato, and P. A. E. M. Janssen, 2011: On the estimation of the kurtosis in directional sea states for freak wave forecasting. J. Phys. Oceanogr., 41, 1484-1497.

Onorato, M., and Coauthors, 2009a: Statistical properties of mechanically generated surface gravity waves: A laboratory experiment in a three-dimensional wave basin. J. Fluid Mech., 637, 235-257.

— , and Coauthors, 2009b: Statistical properties of directional ocean waves, the role of modulational instability in the formation of extreme events. Phys. Rev. Lett., 102, 114502, doi:10.1103/PhysRevLett.102.114502.

Savelyev, I., B. K. Haus, and M. A. Donelan, 2011: Experimental study on wind-wave momentum flux in strongly forced conditions. J. Phys. Oceanogr., 41, 1328-1344.

Segur, H., D. Henderson, J. Carter, J. Hammack, C.-M. Li, D. Pfeiff, and K. Socha, 2005: Stabilizing the Benjamin-Feir instability. J. Fluid Mech., 539, 229-271.

Stolte, S., 1992: Wave breaking characteristics deduced from water staff measurements. Forschungsanstalt der Bendeswehr fur Wasserschall und Geophysik Tech. Rep. FB 1992-4, 21 pp.

Stramska, M., and T. Petelski, 2003: Observations of oceanic whitecaps in the north polar waters of the Atlantic. J. Geophys. Res., 108, 3086, doi:10.1029/2002JC001321.

Toffoli, A., A. V. Babanin, T. Waseda, and M. Onorato, 2010: The maximum steepness of oceanic waves. Geophys. Res. Lett., 37, L05603, doi:10.1029/2009GL041771.

Touboul, J., and C. Kharif, 2010: Nonlinear evolution of the modulational instability under weak forcing and damping. Nat. Hazards Earth Syst. Sci., 10, 2589-2597. 
Trulsen, K., and K. Dysthe, 1992: Action of windstress and breaking on the evolution of a wavetrain. Breaking Waves, M. Banner and R. Grimshaw, Eds., Springer-Verlag, 243-249.

Waseda, T., and M. Tulin, 1999: Experimental study of the stability of deep-water wave trains including wind effects. J. Fluid Mech., 401, 55-84.

— - T. Kinoshita, and H. Tamura, 2009: Interplay of resonant and quasi-resonant interaction of the directional ocean waves. J. Phys. Oceanogr., 39, 2351-2362.
Wu, J., 2000: Bubbles produced by breaking waves in fresh and salt waters. J. Phys. Oceanogr., 30, 1809-1813.

Yuan, Y., L. Han, F. Hua, S. Zhang, F. Qiao, Y. Yang, and C. Xia, 2009: The statistical theory of breaking entrainment depth and surface whitecap coverage of real sea waves. J. Phys. Oceanogr., 39, 143-161.

Zhao, D., and Y. Toba, 2001: Dependence of whitecap coverage on wind and wind-wave properties. J. Oceanogr., 57, 603616. 